\title{
The second-order effect of Earth's rotation on Cryosat-2 fully focused SAR processing
}

\author{
Marcel Kleinherenbrink ${ }^{1} \cdot$ Walter H. F. Smith ${ }^{2} \cdot$ Marc C. Naeije $^{1} \cdot$ D. Cornelis Slobbe ${ }^{3} \cdot$ Peter Hoogeboom $^{3}$
}

Received: 18 January 2019 / Accepted: 17 December 2019 / Published online: 2 January 2020

(c) The Author(s) 2020

\begin{abstract}
We investigated the sensitivity of fully focused SAR (FF-SAR) processing of Cryosat-2 altimeter data to Earth rotation. Earth's rotation causes scatterers at varying cross-track locations to have a different relative velocity with respect to the satellite. This second-order effect of Earth rotation on the phase is currently not corrected for in FF-SAR processing of altimetry data. The difference is largest near the poles, where the satellite flies parallel to the equator. Not correcting for the second-order effect yields a parabolic shape in the counter-rotated phase, which increases with the cross-track distance. Its effect is, however, limited by the time-in-view of the scatterer, which is shorter at the edge of the altimeter footprint, and therefore destructive interference will not occur when using Cryosat-2 data. For Cryosat-2, the only expected effect is a reduction in power and along-track resolution in the waveform tail and in the grating lobes. If the FF-SAR processor focuses on one point, and there is a bright scatterer at another, then there is a residual parabolic phase, whose sign and shape depend on the cross-track distance and whether the signal is left or right of the chosen focal point. In theory, if the viewed scene only has few bright coherent scatterers, then it might be possible to determine the cross-track position of each. In practice, however, natural targets are rarely coherent over the integration time.
\end{abstract}

Keywords Cryosat- $2 \cdot$ SAR $\cdot$ Altimetry $\cdot$ Earth rotation $\cdot$ Focusing $\cdot$ Land contamination

\section{Introduction}

With the launch of Cryosat-2 in April 2010, a new era of satellite radar altimetry began. Cryosat-2 carries the Synthetic aperture radar Interferometric Radar ALtimeter (SIRAL), which is capable of operating in three different modes. In two of the modes, Synthetic Aperture Radar (SAR) and SAR Interferometry (SARIn), radar pulses are transmitted

This study is funded by the Netherlands Organisation for Scientific Research (NWO) through Grant ALW-GO/13-04: Ocean application of the CryoSat-2 SAR mode: preparing for Sentinel-3 and Jason-CS. The manuscript contents do not constitute a statement of policy, decision, or position on behalf of NOAA or the U.S. Government.

Marcel Kleinherenbrink m.kleinherenbrink@tudelft.nl

1 Astrodynamics and Space Missions, Delft University of Technology, Kluyverweg 1, 2629 HS Delft, The Netherlands

2 NOAA Center for Weather and Climate Prediction, 5830 University Research Ct, College Park, MD 20740, USA

3 Geoscience and Remote Sensing, Delft University of Technology, Stevinweg 3, 2628 CN Delft, The Netherlands in bursts. This allows for a coherent processing of 64 pulses (Raney 1998; Wingham et al. 2006). The main advantage of doing so is the increase in the along-track resolution from several kilometers to about $300 \mathrm{~m}$. This increase is required to detect more leads in sea-ice-covered regions (Wingham et al. 2006) and to reduce land-signal waveform contamination in coastal regions (Dinardo et al. 2018). A second advantage is the increase in the number of independent looks, which enhances the precision of the retrieved geophysical parameters (Raney 1998).

The standard processing of Cryosat-2 SAR mode data, known as delay/Doppler, can be described as closed-burst unfocused SAR processing (Raney 2012). Unfocused indicates that the resolution is still larger than the Fresnel zone (Raney 1998), which is about $170 \mathrm{~m}$ for Cryosat-2. In a recent study, Egido and Smith (2017) demonstrated that the altimeter system onboard Cryosat-2 is stable enough to allow for interburst coherent processing. If the full synthetic aperture length of about $2 \mathrm{~s}$ is exploited, the processing is known as fully focused SAR (FF-SAR). Using FF-SAR processing, the along-track resolution is close to the theoretical limit of half the antenna length, which is about $0.5 \mathrm{~m}$. As demon- 
strated by Egido and Smith (2017), the FF-SAR processing has advantages in targeting small lakes, rivers, sea-ice leads and coastal zones. Egido and Smith (2017) also showed that grating lobes occur at approximately every $90 \mathrm{~m}$ along track as a consequence of the closed-burst sampling of Cryosat- 2 . The grating lobes complicate the interpretation and further analysis of the waveforms in scenes with multiple bright targets.

The two Sentinel-3 satellites are also operated in SAR mode, which allows to apply FF-SAR processing to their data as well. Sentinel-3 also applies closed-burst pulsing, which causes grating lobes similar to those of Cryosat-2 (Guccione et al. 2018). Sentinel-6 is scheduled to be launched in 2021, which will be the first SAR altimeter to apply interleaved pulsing. This removes the along-track contamination caused by the grating lobes. However, the pulse repetition frequency is reduced, as compared to Sentinel-3, from 18 to $9 \mathrm{kHz}$, causing aliasing beyond about $1 \mathrm{~s}$ integration time. Crosstrack interferometry is not possible for the Sentinels, as the satellites do not carry a second altimeter antenna.

The FF-SAR processing procedure consists of four steps to counter rotate phases, such that focusing is achieved (Egido and Smith 2017). A range cell migration correction (step 1) ensures that the response from a scatterer is aligned after range compression is applied (step 2). Then, a small residual video phase (RVP) correction (step 3) accounts for quadratic effects of the phase, which is applied in Egido and Smith (2017) for all cross-track positions separately. To focus the signal, thousands of cycles are counter rotated by the relative range phase (RRP) correction (step 4), which is by far the largest correction term and therefore the most sensitive.

To enable a computationally efficient implementation of the RRP, Egido and Smith (2017) provides an equation for the range history. If $R_{i}$ is the minimum range to the scatterer $i$ and $R_{0}$ the minimum range to a nadir target, the range history $R_{i}(\eta)$ is given as:

$$
R_{i}(\eta)=\sqrt{R_{0}^{2}(\eta)+\left(R_{i}^{2}-R_{0}^{2}\right)}
$$

where $\eta$ denotes slow time. This combines a parabolic term $R_{0}^{2}(\eta)$ for a nadir target with an offset, to determine the range history for a scatterer cross-track. We will refer to this small offset as the static geometrical correction. If the FF-SAR calculation is performed to focus on the nadir ground track and the static geometrical correction is applied to points off-track, the squared terms in Eq. 1 produce a correction with even symmetry across track. Barber (1985) shows that in a side-looking SAR system, the effect of Earth rotation on slow time range history depends on cross-track position. Therefore, Earth rotation as a function of cross-track position is commonly taken into account in conventional SAR processing. We find in this paper that the same is true for a nadir-looking altimeter, when the FF-SAR processing is applied.

In this study, we will investigate the validity and applicability of this range estimate for the RRP correction. First, it is demonstrated that the application of Eq. 1 yields a residual parabolic phase in the observed data over a transponder. Second, using a model, based on a spherical Earth model and a circular orbit, a reconstruction of the residual phase is made. The model is applied to varying latitudes to see how the amplitude of the residual range varies geographically. Third, a simplified equation is given to provide more insight into the cross-track range history variations caused by Earth rotation. Eventually, we will discuss the consequences and potential benefits for FF-SAR processing.

\section{Phase history over a transponder}

We argue that the application Eq. 1 can lead to a significant residual phase when a scatterer is not on the ground track. In Egido and Smith (2017), when focusing on a transponder, they accounted for the cross-track location, and they processed the data so that the range $R_{0}$ corresponds to the transponder location. However, if they had put $R_{0}$ on the nadir ground track and used Eq. 1, they would have made an error. To verify this, we counter rotate observed Cryosat-2 SAR mode data of a transponder overpass. If our thesis is true, a remaining parabolic phase is present after the RRP correction, which is related to the cross-track distance from the ground track. However, a parabolic shape in the phase history over a transponder can emerge from other sources, such as an offset in the carrier frequency, deramping pulse datation biases and incorrect application of FF-SAR processing. For comparison, we therefore model deramped echoes using the equations for the phase of the deramped signal in Egido and Smith (2017) with as input the satellite emphemerides found in the data file. After that, the four FF-SAR processing steps as listed above are applied.

Figure 1a shows the counter-rotated phase against slow time of the modeled transponder signal using Eq. 1 for the RRP and the RVP corrections, rather than at the actual cross-track position of the transponder, like Egido and Smith (2017). We used the overpass on 04-08-2015, for which the cross-track distance to the transponder is $2.8 \mathrm{~km}$. Since the observed counter-rotated phase in panel B shows similar behavior, the previously described other sources on the phase have been removed properly or are negligible. In panels $\mathrm{C}$ and $\mathrm{D}$, the ranges for the RRP correction are computed directly using the transponder locations and the satellite burst locations in the Earth-Centered Earth-Fixed (ECEF) reference frame. The flattening of the phase with this approach has two implications. First, for the RVP correction it is sufficient to use the ranges as described by Eq. 1, but for the RRP it 
Fig. 1 Focused phase over the Svalbard transponder expressed in one-way range equivalent. Modeled (a) and observed (b) counter-rotated phase using the ranges as in Eq. 1 with $R_{0}$ at the point on the nadir ground track closest to the transponder. Modeled (c) and observed (d) counter-rotated phase using the ranges as in Eq. 1 with $R_{0}$ at the actual transponder position.

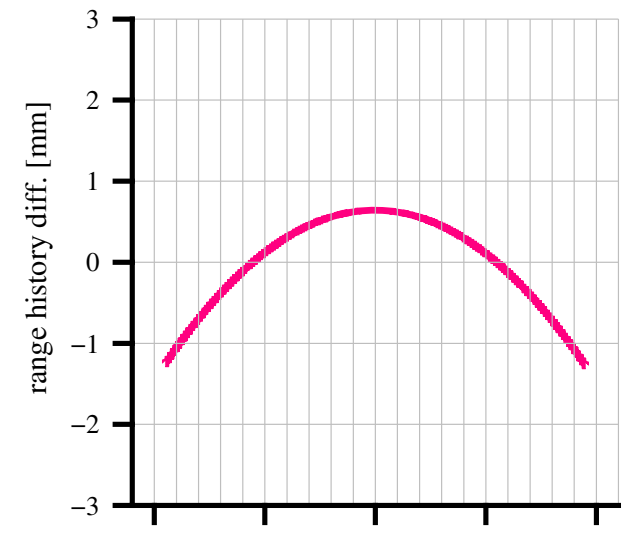

A

B
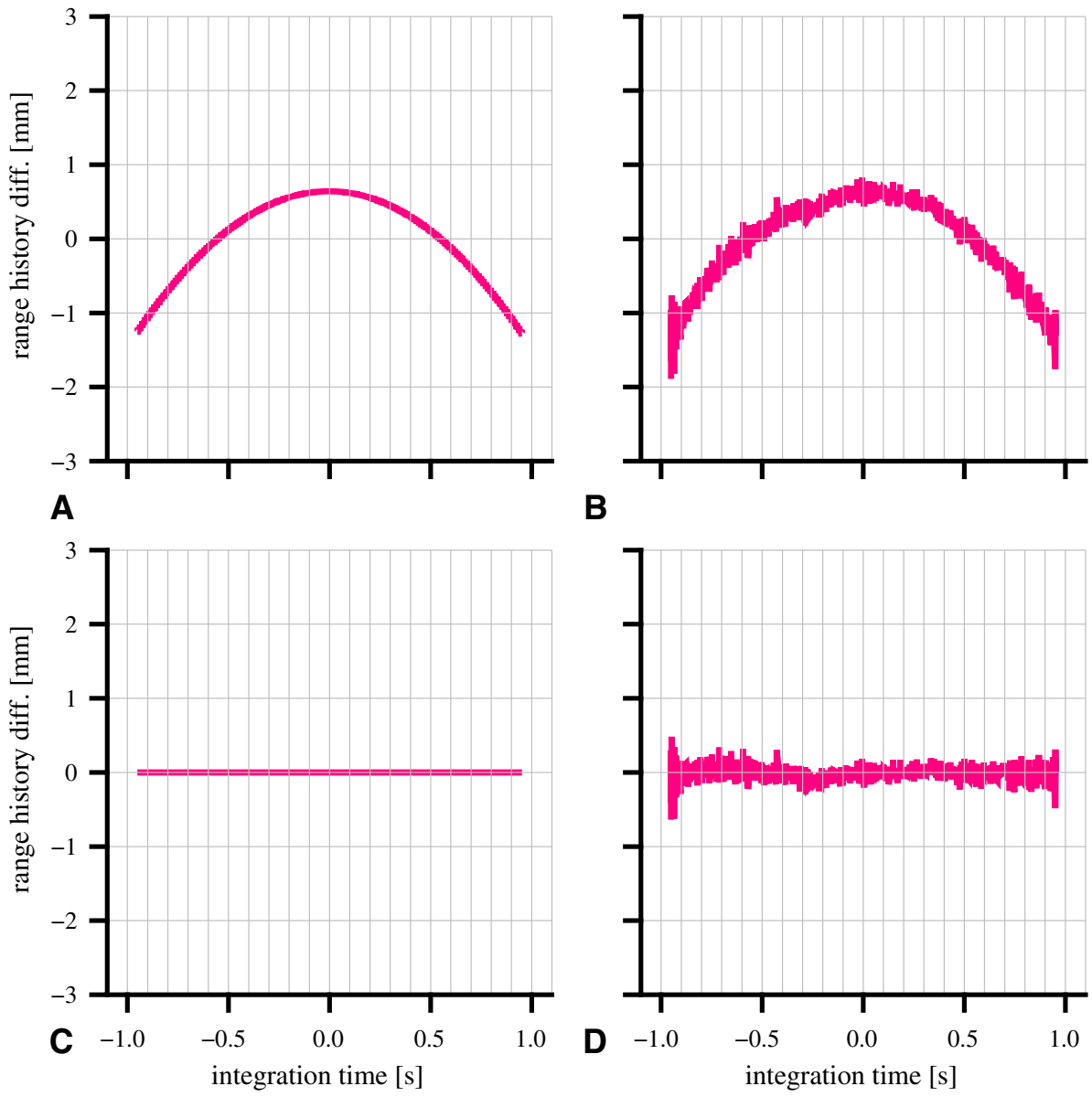

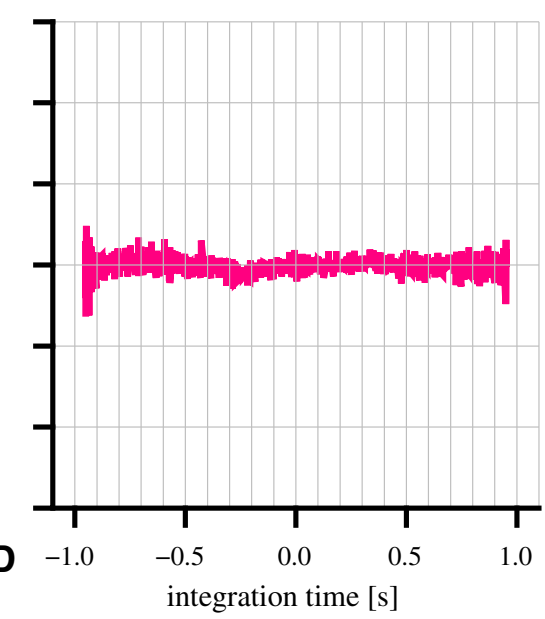

is not, because we find a remaining phase corresponding to a range variation of more than a millimeter. Second, there is an additional contributing effect to the relative ranges, which is not accounted for in Eq. 1 and it relates to the cross-track position of the scatterer.

Since the position of the satellite pulses in the EarthCentered (EC) frame is independent of the cross-track position of the scatterer, the culprit is a relative difference in along-track position of the scatterers as a consequence of Earth rotation. A careful analysis of the second-order range effects due to Earth rotation was already performed by Barber (1985) for side-looking orbital SAR systems, but Egido and Smith did not discuss it, and Eq. 1 supposes that it is negligible in a nadir-looking FF-SAR altimeter. Note that the scatterer position is changing in an EC (but not fixed) frame during the 2-s integration time as the Earth rotates. At different cross-track locations, the latitude coordinate is not equal, and from the satellite perspective, the relative velocity of the scatterer is therefore different. At the northernmost point of Cryosat-2, which corresponds to a ground-track location with a latitude of 88 degrees, the cross-track positions of the scatterer are North and South of the track. The radius toward the rotation axis of the Earth is smaller for the northward scatterer, and therefore the translation of it during 2-s integration time is smaller than for the southward scatterer. This effect is not captured by Eq. 1 .

\section{Range history modeling}

This section describes a model to analyze the effect of Earth rotation on the phase history for cross-track scatterers. The model assumes that the Earth is spherical and the orbit is circular. With the model, it is possible to approximate the magnitude of the effect of Earth rotation on the phase history as a function of cross-track distance and latitude.

\subsection{Ground-track simulation}

A simulation of the ground track based on a dual-axis spiral method is performed to estimate the effect of a rotating Earth on the estimated ranges. The equations in this section have been derived from Wertz et al. (2001). With this approach, we explain the largest part of remaining parabolic phase over the Svalbard transponder. The two axes required for the simulation are the Earth's rotation axis and the orbit rotation axis. 
Since Cryosat-2 has an inclination of $92^{\circ}$, the poles of the two axes have latitudes of $90^{\circ}$ and $-2^{\circ}$, respectively. The longitude $\alpha_{O}$ of the orbit pole is drifting over time $t$ due to Earth's rotation, such that

$\alpha_{O}=\alpha_{O, 0}-\omega_{E} t$,

where we set the initial value of the longitude $\alpha_{O, 0}$ to $-90^{\circ}$ and $\omega_{E}$ is the angular velocity of the Earth. The counterclockwise angle $\phi_{S}$ around the orbit axis is computed as

$\phi_{S}=270^{\circ}+\phi_{S, 0}+n t$,

where $n$ is the angular velocity of the satellite and $\phi_{S, 0}$ is the initial value for the angle around the orbit axis. By setting $\phi_{S, 0}=0^{\circ}$ in a polar orbit, the satellite crosses the equator in an ascending track and by setting $\phi_{S, 0}=90^{\circ}$, the satellite is at the maximum latitude, i.e., the inclination of the orbit. The latitude $\delta$ of the satellite in the ECEF frame is then computed as

$\delta=90^{\circ}-\arccos \left(\sin (i) \cos \left(\phi_{S}\right)\right)$,

with $i$ the inclination of Cryosat-2's orbit. The difference in longitude $\Delta \alpha$ between the satellite and the orbit pole is computed with

$\Delta \alpha=-H\left(\phi_{S}\right) \arccos \left(\frac{-\tan (\delta)}{\tan (i)}\right) \bmod _{360}$,

where mod represents modulo and

$$
\begin{aligned}
& H\left(\phi_{S}\right)=+1, \quad 0 \leq \phi_{S}<180 \\
& H\left(\phi_{S}\right)=-1, \quad 180 \leq \phi_{S}<360 .
\end{aligned}
$$

Lastly, the longitude of the satellite $\alpha$ is determined as

$\alpha=\left(\alpha_{O}+\Delta \alpha\right) \bmod _{360}$.

The heading of the satellite is computed using the coordinates of two consecutive epochs with the MATLAB geodetic toolbox. The arc lengths between nadir and cross-track scatterers are used in combination with the ground-track heading to determine their respective longitudes and latitudes. By converting the satellite, the nadir, and the scatterer locations to Cartesian coordinates, the ranges between the satellite within the 2-s integration time and the scatterers are easily computed. A MATLAB code is provided as supplementary material.

\subsection{Conceptual model of the range history}

While the dual-axis spiral gives a good approximation of the range history, the conceptual model is setup to provide more insight into the geometry of the Earth rotation effect on the range. The conceptual model is, however, still accurate to several tenths of millimeter at $3 \mathrm{~km}$ cross-track. As the range history difference is largest near the poles, we first design a conceptual model at the pole. To support the interpretation, Fig. 2 shows the relative positions of the bursts and two crosstrack scatterers.

Similar to the dual-axis spiral, a spherical Earth is used, but we approximate the satellite orbit by a straight line. The satellite is assumed to fly in a westerly direction, parallel to the equator at 88 degrees north. The origin of the local reference is the nadir location of the satellite at $t=0$ seconds, while the coherent processing time runs approximately from -1 to $1 \mathrm{~s}$. We take the $x$-, $y$ - and $z$-axis as the cross-track direction, along-track direction and up directions, respectively. The $y$-coordinate changes from approximately -7.5 to 7.5 $\mathrm{km}$ during the coherent processing time, due to the satellite velocity and the motion of the scatterer. Using the listed approximations, we can compute the range $R_{0}(t)$ from the satellite to the nadir location with

$R_{0}(t)=\sqrt{\left(y_{0}(t)+\Delta y_{0}(t)\right)^{2}+z^{2}}$,

where $\left(0, y_{0}(t)+\Delta y_{0}(t), z\right)$ are the satellite coordinates in the local reference frame and where $\Delta y_{0}(t)$ is the contribution of the scatterer's motion due to Earth's rotation. We assume that the rotation of the Earth only contributes to a translation in the along-track direction. The Earth rotates in the easterly direction, which implies that at $t=-1$ and $t=1$ $\mathrm{s}$, the satellite is slightly further away from the scatterer than when the Earth would not rotate. The value for $\Delta y_{0}(t)$ can be computed as

$\Delta y_{0}(t)=\omega_{E} t \cdot \cos \left(\delta_{0}\right)$,

with $\delta_{0}$ the latitude of the nadir location at $t=0$ seconds.

A second reference frame is setup, with the cross-track scatterer location as the origin (see right panel of Fig. 2). If we approximate the cross-track strip as a straight line, this implies that the $x$-coordinate $x_{s}$ of the satellite changes and the contribution of the scatterer motion $\Delta y_{s}(t)$ changes, due to a change in latitude. The range $R_{S}(t)$ from the satellite to the scatterer is described as

$R_{S}(t)=\sqrt{x_{s}^{2}+\left(y_{0}(t)+\Delta y_{s}(t)\right)^{2}+z^{2}}$,

where $\left(x_{s}, y_{0}(t)+\Delta y_{s}(t), z\right)$ are the satellite coordinates in the scatterer reference frame and the contribution of the scatterer motion is given by:

$\Delta y_{s}(t)=\omega_{E} t \cdot \cos \left(\delta_{s}\right)$, 


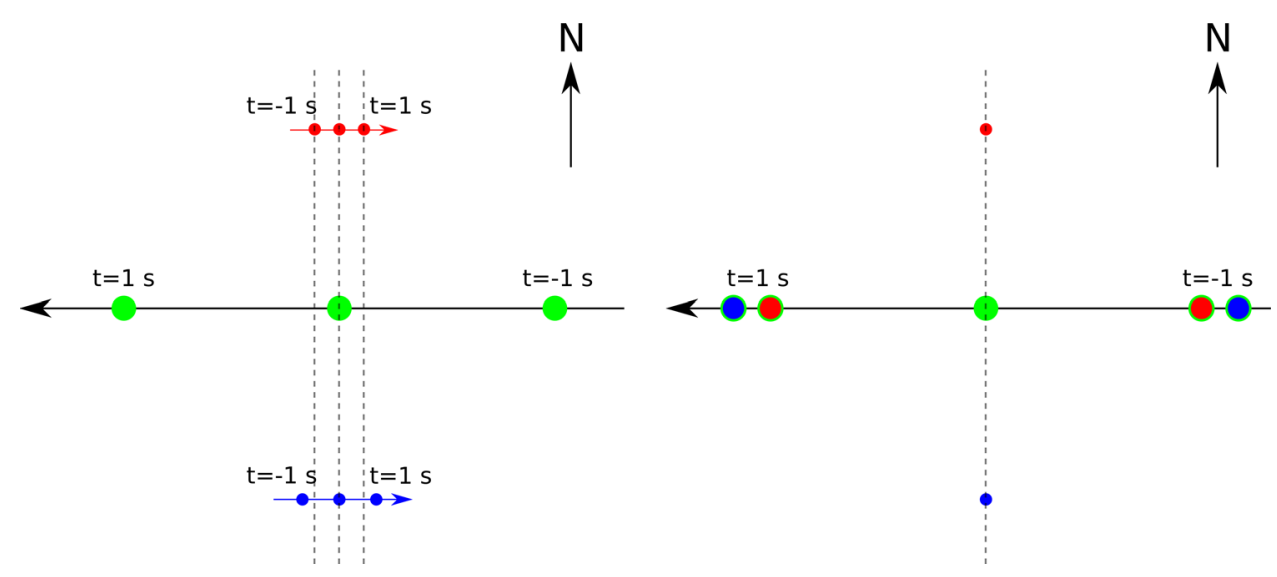

Fig. 2 Illustration of the conceptual model. In the Earth-centered nonfixed reference system (left), the first burst (green) is received at east of the last burst as Cryosat-2 is in a retrograde orbit. Due to Earth rotation, scatterers on the northside (red) and southside (blue) move at different

where $\delta_{s}$ is the latitude of the scatterer at $t=0$ seconds. If we ignore the contribution of a rotating Earth, the only range difference between a nadir and a cross-track scatterer is the contribution of $x_{s}$. This amounts to several millimeters in range at the edge of the antenna beam (Egido and Smith 2017) and is adjusted for by Eq. 1. On a rotating Earth, however, there is a difference between $\Delta y_{0}(t)$ and $\Delta y_{s}(t)$, and we will demonstrate that this can become larger than the static geometrical effect. The contributions of $\Delta y_{0}(t)$ and $\Delta y_{s}(t)$ are scaled by the absolute value of the sine of the satellite heading and therefore decrease toward the equator.

\section{Discussion of the results}

As a reference, we assume that the scatterer is located at 3 $\mathrm{km}$ cross-track. Using the conceptual model of Sect. 3.2, an approximation can be made for smaller or larger cross-track distances. Figure 3 shows range history difference between nadir locations and cross-track locations at the equator and at a latitude of $88^{\circ}$. Note that for all the plots, a constant value of approximately $6 \mathrm{~m}$ is removed, which is the difference in range between a scatterer at $3 \mathrm{~km}$ cross-track and nadir at $t=0 \mathrm{~s}$

Panel A shows that the range history difference is maximally $0.4 \mathrm{~mm}$ if the Earth is not rotating. This number is similar for scatterers on the right and the left side of nadir (panels A and C) and independent on latitude. In the conceptual model, for a non-rotating Earth, this implies that $\Delta y_{0}(t)=\Delta y_{s}(t)=0$, and therefore it is only affected by the cross-track scatterer distance $x_{s}$.

In panels $\mathrm{B}$ and $\mathrm{D}$, the range history difference of the orange curve does not change significantly, which is expected as the contributions $\Delta y_{0}(t)$ and $\Delta y_{s}(t)$ decrease toward the equator. There is an additional translation in the $\mathrm{x}$-direction, velocities. In the scatterer reference frames (right), the relative distance between the first burst and the last is larger for scatterers south of the ground track (blue) than for scatterers north of the ground track (red)

but this will not contribute significantly, because of the small angles. At $88^{\circ}$, the satellite moves against the direction of the Earth's rotation, which implies that the satellite is at $t=-1$ and $t=1 \mathrm{~s}$ about $16 \mathrm{~m}$ further away from the focusing strip. Because the nadir latitude and the scatterer latitude differ, a difference of $\Delta y_{s}(1)-\Delta y_{0}(1)=0.2 \mathrm{~m}$ is introduced, while the difference of $\Delta y_{s}(0)-\Delta y_{0}(0)=0 \mathrm{~m}$ if the scatterer is located on the north side of the track. For a scatterer located $3 \mathrm{~km}$ cross-track, this implies a $2.7 \mathrm{~mm}$ difference in range history (panel B).

The opposite happens when the scatterer is located south of the track (panel D). This is a consequence of a difference in Earth's surface velocity south of track with respect to the north side, due to the larger distance from the Earth's rotation axis. The signal, however, is with $1.9 \mathrm{~mm}$ smaller in amplitude compared to the scatterer north of the track, because the total signal is balanced around the $0.4 \mathrm{~mm}$ caused by the additional range due to the cross-track position $x_{s}$. If the RRP correction is applied as in Eq. 1, the $0.4 \mathrm{~mm}$ is compensated for, so for scatterers located at $3 \mathrm{~km}$ cross-track on both sides a residual phase parabola of $2.3 \mathrm{~mm}$ is present, but they are of opposite sign for left and right scatterers.

Panel A in Fig. 4 shows the amplitude of the difference in phase history between a nadir and a cross-track scatterer as a function of latitude. The signals coming from left and right are mirrored around the equator, which is expected since a scatterer on the right is further away from the equator at the north pole than at the south pole due to the retrograde motion of the Cryosat orbit. If a correction for the static geometrical range change (pink line) of $0.4 \mathrm{~mm}$ is applied, the phase histories left and right are practically mirrored over the ground track at any latitude. The largest signals are, as discussed earlier, found near the poles, where the satellite flies parallel to the Equator. 
Fig. 3 Modeled range history difference between a scatterer at nadir and at $3 \mathrm{~km}$ cross-track. In orange, the phase history difference at the equator and in blue at $88^{\circ}$ latitude. a Difference with right of nadir without Earth rotation. $\mathbf{b}$ Difference with right of nadir with Earth rotation. c Difference with left of nadir without Earth rotation. d Difference with left of nadir with Earth rotation

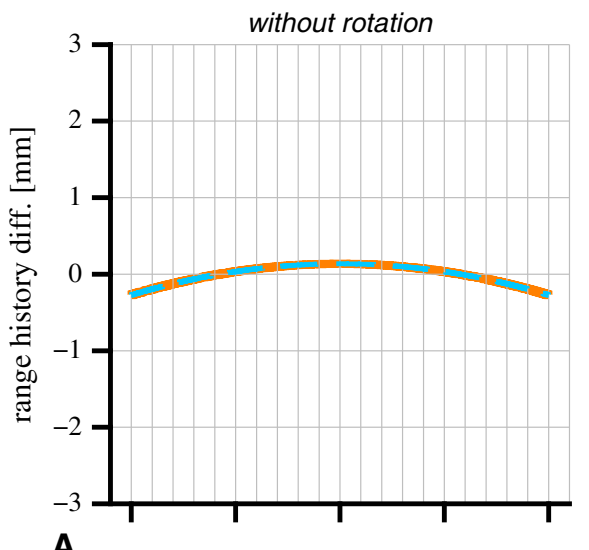

\section{A}

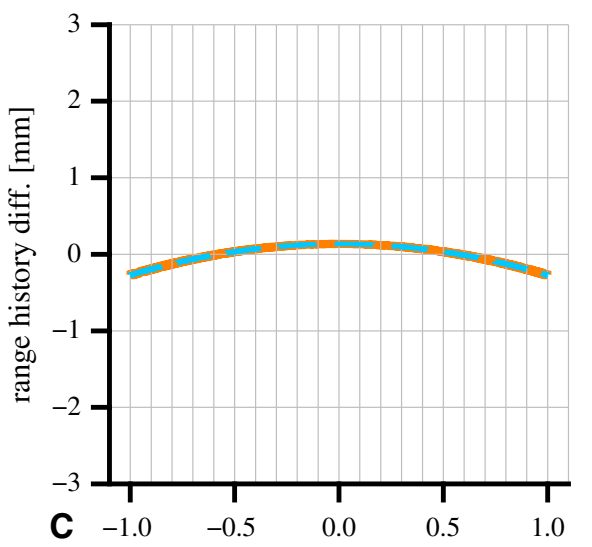

integration time $[\mathrm{s}]$

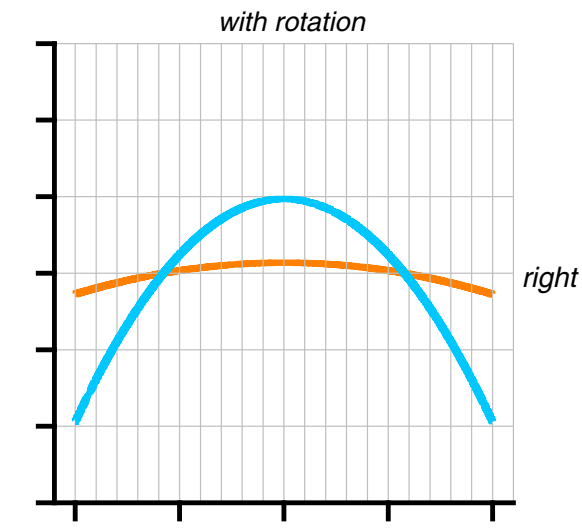

B

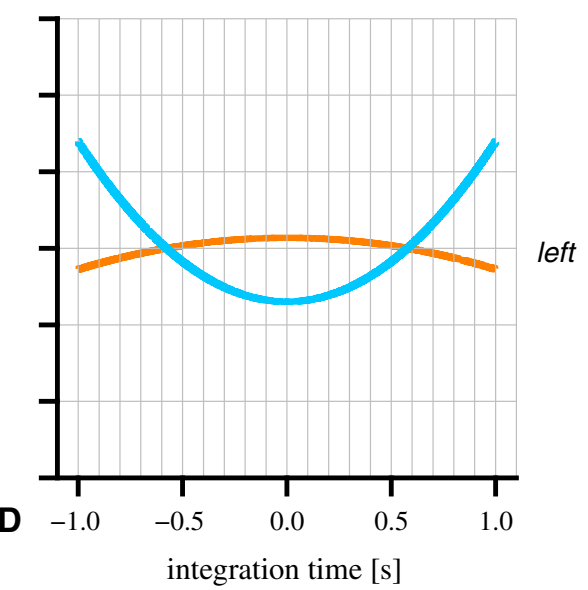



Fig. 4 Modeled amplitudes of the range difference. In orange, the amplitude for a scatterer at the right side of the ground track and in blue on the left side. Positive values indicate a positive parabola, and negative values indicate a negative parabola. The pink line shows the geometrical effect on the range without Earth rotation for which is

If the cross-track angle becomes larger, the phase history differences also become larger, which can cause destructive interference. However, we have to take into consideration that the effective coherent processing time decreases because of two reasons. First, if the onboard tracker correctly follows

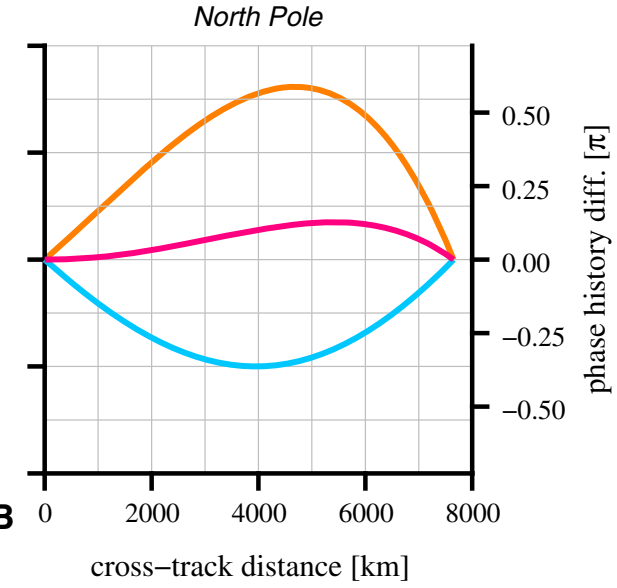

compensated by Eq. 1. Destructive interference occurs at $\frac{\pi}{2}$ radians. a Amplitude of the signal at $3 \mathrm{~km}$ cross-track as a function of latitude. $\mathbf{b}$ Amplitude of the signal at the maximum latitude of $88^{\circ}$ as a function of cross-track distance

the nadir path, the cross-track signals will appear late in the waveform. As discussed by Guccione et al. (2018), in SAR mode, the $60 \mathrm{~m}$ range window is too short to support a 2-s integration time. If the surface is not flat and the scatterer is close to tracking point of the altimeter, it supports a longer 
Fig. 5 PTR based on FF-SAR processing of Cryosat-2 SAR data over Svalbard using focal points set at 0.0 (a), 2.8 (b) and $4.0(\mathbf{c}) \mathrm{km}$ from the cross-track location of the transponder. The integration time is kept constant at $1.8 \mathrm{~s}$. In the panel d, the along-track cross sections of the main lobes, including one with a focal point at $5.6 \mathrm{~km}$ from the transponder, which is the cross-track distance for which Sentinel-3 support $1.8 \mathrm{~s}$ integration time
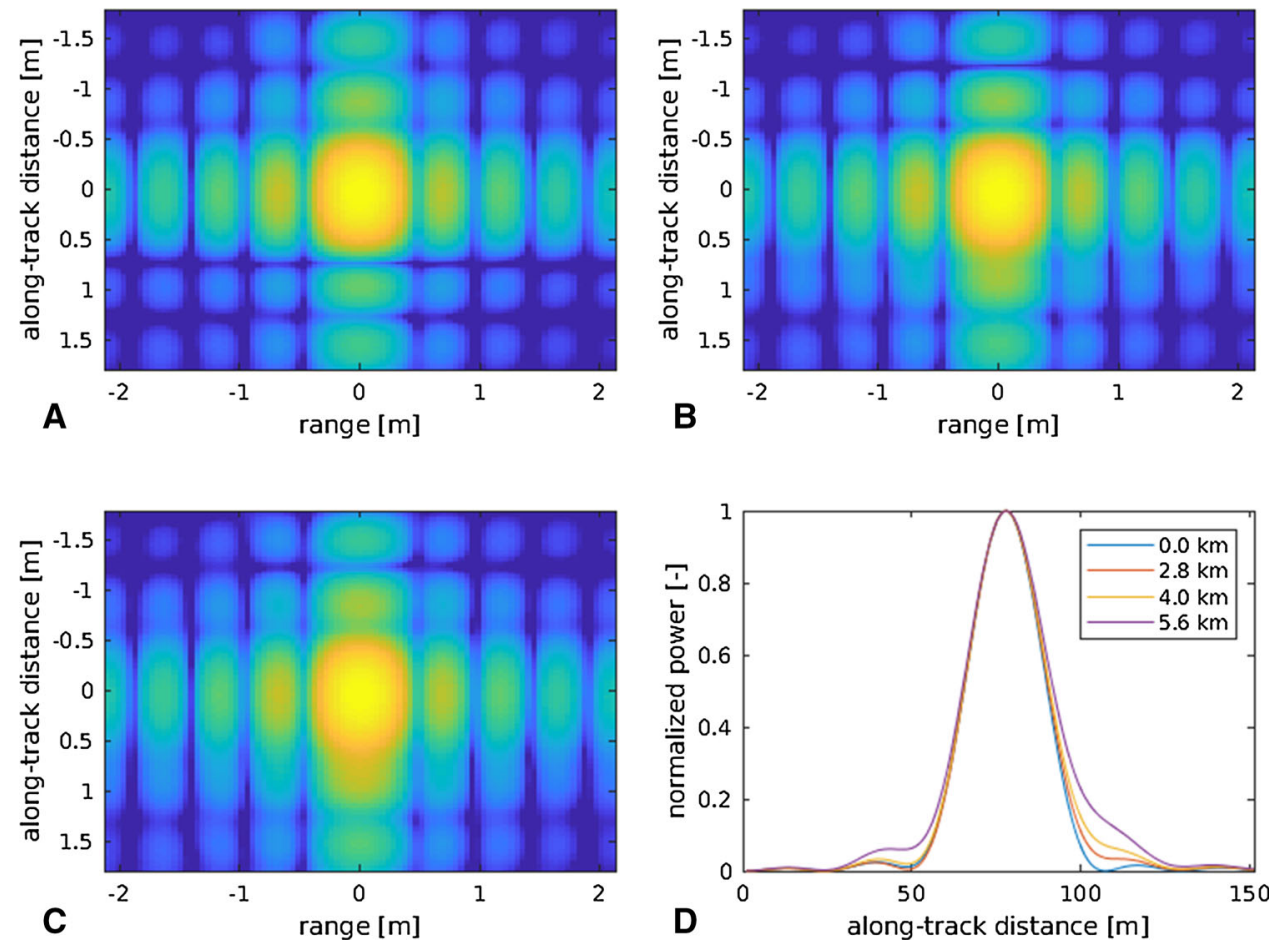

coherent processing time. In SARIn mode, the range window is larger, so cross-track scatterers are visible over the whole overpass.

The second limiting factor, the elliptical footprint shape, limits the integration time even in SARIn mode. Therefore, we compute the effective integration time based on the footprint shape (Appendix A) and use it to constrain the amplitude of the range history difference as a function of cross-track distance (panel B). By taking the coherent processing time into account, the maximum range history difference with nadir occurs between 4 and $5 \mathrm{~km}$ cross-track. Once corrected for the static geometrical effect as with Eq. 1, the maximum amplitude drops below $2.8 \mathrm{~mm}$ (equivalent to a $\frac{\pi}{2}$ radian phase difference), and no destructive interference will occur. Note that since the range history is parabolic, a slight increase in integration time causes a large phase difference. Due to its $19 \mathrm{~km}$ (based on a beamwidth of $1.35^{\circ}$ ) footprint, which is 4-5 km larger than that of Cryosat-2, Sentinel-3 is more sensitive to Earth rotation. Sentinel-6 is even more sensitive as it supports an even longer integration time, but we have to consider aliasing caused by the lower PRF of $9 \mathrm{kHz}$. To avoid aliasing, the coherent processing time should be limited to approximately $1 \mathrm{~s}$. However, a longer coherent processing time may be useful in case of a limited number of bright spots in the scene (so that aliasing from along-track targets is limited), or when deconvolution techniques can be applied. In that case substantial deconstructive interference is expected, since the range window of the raw data is larger than that of Sentinel-3, and the satellite orbits at a higher altitude.
Even though for Cryosat-2 the signal does not suffer from destructive interference, the power in the waveform reduces and the main lobe of the point-target response widens, because the phases are not perfectly aligned. In Fig. 5, the widening of the main lobe is investigated by moving the focal point away from the transponder. We use the same pass as in the other sections for which the transponder is located 2.8 $\mathrm{km}$ left of the ground track. For an integration time of 1.8 $\mathrm{s}$, corresponding to an along-track resolution of $59 \mathrm{~cm}$, the increase in the main lobe width is about $1 \mathrm{~cm}$ if the focal point is at the nadir location. Cryosat supports a $1.8 \mathrm{~s}$ integration time at $4 \mathrm{~km}$ across track, which is close to where the parabolic phase residual reaches its maximum amplitude. By moving the focal point $4 \mathrm{~km}$ from the transponder, this results in a widening of the main lobe of $2 \mathrm{~cm}$. Sentinel-3 supports a $1.8 \mathrm{~s}$ integration time at $6 \mathrm{~km}$ across track, which results in a widening of about 5-6 cm (or nearly 10\%). Using a single nadir focal point results effectively in a reduced resolution in the waveforms tails, and therefore consecutive waveforms are not fully independent.

The change in power has consequences for several applications. For power calibrations with a transponder based on FF-SAR-processed data, it is required to use the exact ranges and not an estimate based on Eq. 1. Besides changes in the power of the main lobe, also the power of the grating lobes changes as already demonstrated for the WK algorithm (Guccione et al. 2018). The removal of grating lobes using deconvolution techniques (Egido and Smith 2017) is therefore only straightforward in case of nadir targets if Eq. 1 
Fig. 6 Orange lines represent and the corresponding waveform (right) over the transponder by using the exact ranges for the RRP correction. The blue lines represent the situation in which we assumed that the transponder was on the other side of the ground track. No correction for the antenna pattern is applied the counter-rotated phase (left)
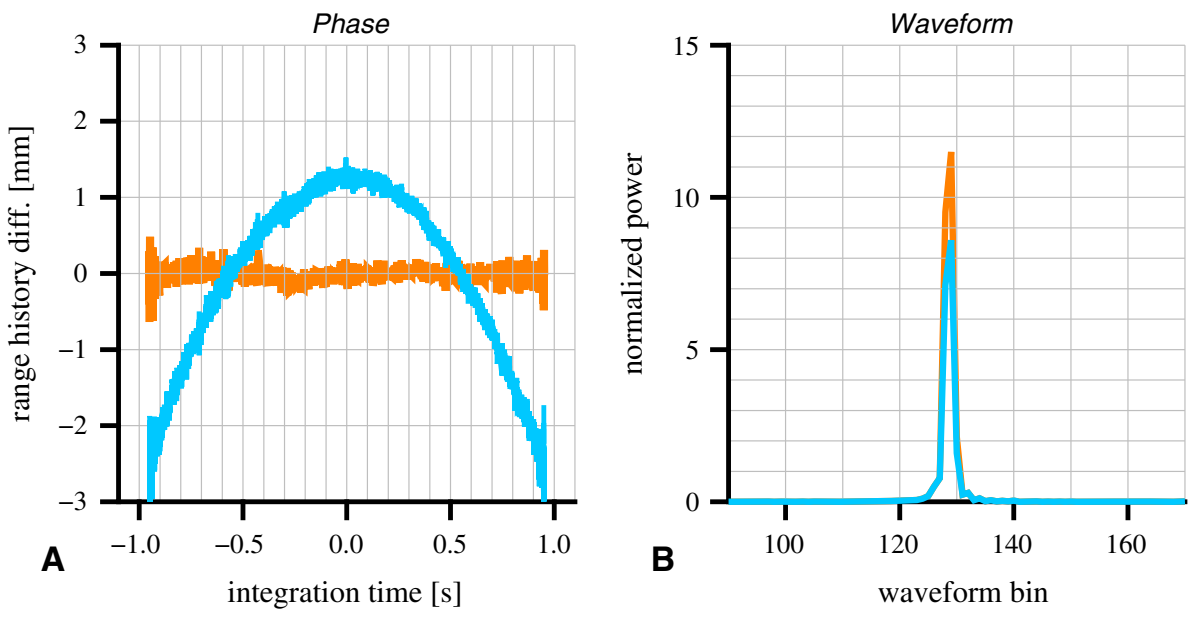

Fig. 7 Normalized power radargram after the RCMC correction for the Svalbard transponder (a) and a bright natural target (b) in the Netherlands. The transponder is visible over the whole integration time, while the natural target only for a fraction. Signals from several along-track scatterers on a nearly flat surface cause the parabolic pattern in panel b

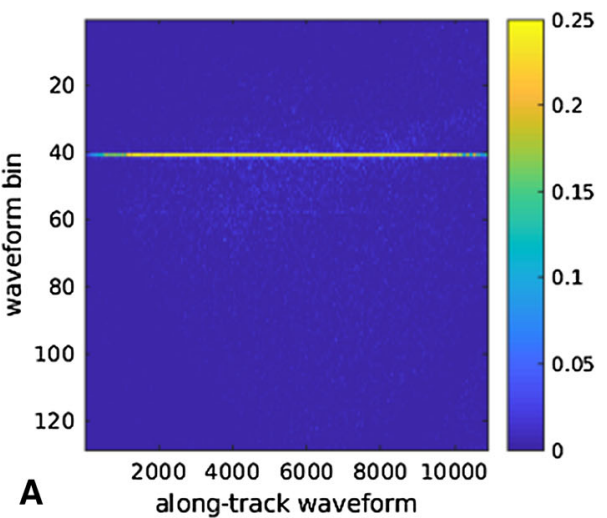



is used. As shown in Fig. 4, the effect of differential earth rotation on residual phase has antisymmetry (odd symmetry) with respect to left-right displacement across track, which may be exploited as follows. In Fig. 6 panel A, the counterrotated phases over the transponder are shown based on the exact ranges, which results in a flat phase history. Again, the transponder is located at $2.8 \mathrm{~km}$ cross-track. If we assume the transponder is on the other side of the track and use the range history of that location to counter rotate the phases, a large parabolic phase is left and the transponder signal becomes unfocused. The accompanying waveform power is shown in panel B, where the unfocused waveform has 20-30\% less power.

In theory, the reduction in power by bringing targets outof-focus can be exploited. First, by focusing on one side of the ground track, it is possible to mitigate the influence of targets on the other side. In case a satellite is parallel to a shoreline, the waveforms might be partially cleaned from land contamination. This requires the scatterers to be coherent and visible over a large fraction of the integration time of 2 s. Fig. 7 shows the signal of a natural target (in this case a pond) in a radargram. The reflection from the pond is only visible in a fraction of the $\sim 10,000$ waveforms used in the 2-s integration time. This limits the application of the proposed method, because most contaminating targets show similar behavior in terms of visibility as the pond. Additionally, the along-track location of the contaminating scatterer would have to be determined accurately to reduce the power of its signal in the waveform. Second, the Cryosat- 2 data can be processed twice by focusing on both sides. Based on the power, it can be determined on which side of the ground track a scatterer is located. At high latitudes, the scatterer location might even be determined unambiguously, since the phase history and the range depend in a different way on the height and cross-track location of the scatterer. Again, it would require scatterers to be visible and coherent over the integration time of $2 \mathrm{~s}$.

\section{Conclusions}

This study addressed the uncorrected second-order effect of Earth rotation on the range for off-nadir scatterers, which affect the FF-SAR processing of satellite radar altimetry data. Earth rotation primarily affects the residual range phase (RRP) correction, which is the largest phase counter rotation to be applied in the FF-SAR process. The effect of Earth rotation becomes larger as the cross-track distance to the 
scatterers increases, and it is opposite sign for scatterers left and right of the ground track. Destructive interference occurs at $\frac{\pi}{2}$ radians, corresponding to 2.8 -mm one-way range history difference. The footprint, however, reduces the coherent processing time for cross-track scatterers. In case of Cryosat-2, destructive interference will not occur if we apply Eq. 1, but a weakening of the power from cross-track signals is present.

Even though destructive interference does not occur if Earth rotation is not taken into account, there are several consequences. The power of grating lobes, which occur due to the closed-burst mode pulsing, degrades, which decreases the polluting signal of bright targets, but will not be eliminated. However, as it is more difficult to determine the magnitude of the grating lobes, a deconvolution algorithm is not straightforward. Additionally, the power of the main lobe changes at off-nadir targets, because they are not exactly in focus. For natural targets, the power change is limited as they are rarely visible and coherent during more than a fraction of a second. For calibrations over transponders, certainly at high latitudes like Svalbard, this should be taken into account.

The left-right difference in phase history caused by Earth rotation provides also additional opportunities. By focusing on targets right of the ground track, targets on the left of the ground track are out-of-phase, which causes their power in the waveform to reduce. This makes the theory possible to decontaminate waveforms or to determine the cross-track location of scatterers. However, natural scatterers are rarely visible and coherent over the 2-s integration time at this wavelength. The practical application is therefore limited to a small number of targets.

Author contributions MK conceived the idea. MK and WS performed the computations. MN supervised the study. MK wrote the manuscript. All authors read the manuscript, provided suggestions and contributed to the discussion of the results.

Data availability After registration, the CryoSat- 2 can be accessed via the ESA FTP server ftp://science-pds.cryosat.esa.int.

Open Access This article is licensed under a Creative Commons Attribution 4.0 International License, which permits use, sharing, adaptation, distribution and reproduction in any medium or format, as long as you give appropriate credit to the original author(s) and the source, provide a link to the Creative Commons licence, and indicate if changes were made. The images or other third party material in this article are included in the article's Creative Commons licence, unless indicated otherwise in a credit line to the material. If material is not included in the article's Creative Commons licence and your intended use is not permitted by statutory regulation or exceeds the permitted use, you will need to obtain permission directly from the copyright holder. To view a copy of this licence, visit http://creativecomm ons.org/licenses/by/4.0/.

\section{Appendix A}

The effective integration time is computed by assuming that the footprint describes a perfect ellipse. For this, we use con-

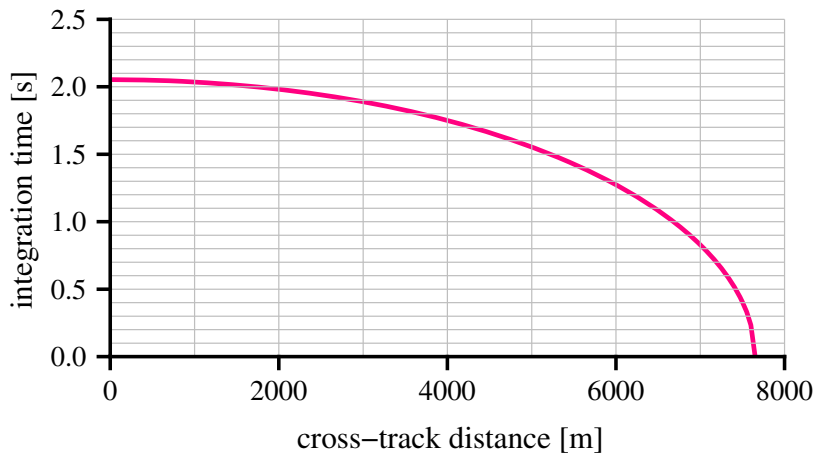

Fig. 8 Estimated effective integration time as a function of cross-track distance

stant values of $13.8 \mathrm{~km}$ and $15.3 \mathrm{~km}$ for the along-track and cross-track diameters of the beam-limited footprint, respectively (Scagliola 2013). The $y$-coordinate $y_{s}\left(\frac{t_{I}}{2}\right)$ of a point on the ellipse is connected to the cross-track distance $x_{s}$ by:

$$
\left(\frac{x_{s}}{a}\right)^{2}+\left(\frac{y_{s}\left(\frac{t_{I}}{2}\right)}{b}\right)^{2}=1 \text {, }
$$

where $a, b$ are the semi-minor and semi-major axis of the ellipse, respectively, $t_{I}$ is the effective integration time, and $\frac{t_{I}}{2}$ is the time to pass half of the footprint. This equation is rewritten as a function of the $y$-coordinate, such that:

$y_{s}\left(\frac{t_{I}}{2}\right)=b \sqrt{1-\left(\frac{x_{s}}{a}\right)^{2}}$.

The ground distance over which the satellite travels over the time a scatterer is in view can be computed as $2 y_{s}\left(t_{I} / 2\right)$. The distance of the satellite in orbit is slightly larger and must therefore be adjusted for height $H$ of the orbit. By assuming a spherical Earth and a circular orbit, the half distance of the satellite traveled is given as:

$y_{b}\left(\frac{t_{I}}{2}\right)=\frac{R_{E}+H}{R_{E}} y_{s}\left(\frac{t_{I}}{2}\right)$,

from which the integration time can be computed with the satellite velocity $V$, such that.

$t_{I}=\frac{2 y_{b}\left(\frac{t_{I}}{2}\right)}{V}$

By computing the integration time for varying cross-track distances, this leads to the result in Fig. 8. At nadir, the effective integration time is slightly over $2 \mathrm{~s}$. A 2-s integration time yields an along-track resolution of approximately 0.5 $\mathrm{m}$. At $5 \mathrm{~km}$ cross-track, the integration time is still $1.5 \mathrm{~s}$, but at larger cross-track distance, it rapidly decays to zero. Above $7 \mathrm{~km}$ cross-track, the integration time is less than 1 
$\mathrm{s}$, and the FF-SAR algorithm therefore yields an along-track resolution worse than $1 \mathrm{~m}$.

\section{References}

Barber BC (1985) Theory of digital imaging from orbital syntheticaperture radar. Int J Remote Sens 6(7):1009-1057. https://doi.org/ 10.1080/01431168508948262

Dinardo S, Fenoglio-Marc L, Buchhaupt C, Becker M, Scharroo R, Fernandes MJ, Benveniste J (2018) Coastal sar and plrm altimetry in german bight and west baltic sea. Adv Space Res 62(6):13711404. https://doi.org/10.1016/j.asr.2017.12.018

Egido A, Smith WH (2017) Fully focused SAR Altimetry: theory and applications. IEEE Trans Geosci Remote Sens 55(1):392-406. https://doi.org/10.1109/TGRS.2016.2607122

Guccione P, Scagliola M, Giudici D (2018) 2D Frequency domain fully focused SAR processing for high PRF radar altimeters. Remote Sens. https://doi.org/10.3390/rs10121943

Raney RK (1998) The delay/Doppler radar altimeter. IEEE Trans Geosci Remote Sens 36(5):1578-1588. https://doi.org/10.1109/ 36.718861

Raney RK (2012) Cryosat SAR-mode looks revisited. IEEE Geosci Remote Sens Lett 9(3):393-397. https://doi.org/10.1109/LGRS. 2011.2170052

Scagliola M (2013) CryoSat footprints. Aresys Technical Note, 1.1, SAR-CRY2-TEN-6331
Wertz JR, Meissinger HF, Newman LK, Smit GN (2001) Mission geometry: orbit and constellation design and management-spacecraft orbit and attitude systems. In: El Segundo JRW (ed) Mission geometry: orbit and constellation design and management-spacecraft orbit and attitude systems. Space technology library, vol 13. Kluwer Academic Publishers, Boston

Wingham DJ, Francis CR, Baker S, Bouzinac C, Brockley D, Cullen R, De Chateau-Thierry P, Laxon SW, Mallow U, Mavrocordatos C, Phalippou L, Ratier G, Rey L, Rostan F, Viau P, Wallis DW (2006) CryoSat: a mission to determine the fluctuations in Earth's land and marine ice fields. Adv Space Res 37(4):841-871. https://doi. org/10.1016/j.asr.2005.07.027 